\title{
Beyond group-level explanations for the failure of groups to solve hidden profiles: The individual preference effect revisited
}

Group Processes \& Intergroup Relations 13(5) 653-671

(C) The Author(s) 2010 Reprints and permission: http://www. sagepub.co.uk/journalsPermission.nav DOI: $10.1177 / 1368430210369143$ gpir.sagepub.com

(S)AGE

\author{
Nadira Faulmüller, ${ }^{1}$ Rudolf Kerschreiter, ${ }^{2}$ Andreas \\ Mojzisch, ${ }^{1}$ and Stefan Schulz-Hardt ${ }^{1}$
}

\begin{abstract}
The individual preference effect supplements the predominant group-level explanations for the failure of groups to solve hidden profiles. Even in the absence of dysfunctional group-level processes, group members tend to stick to their suboptimal initial decision preferences due to preference-consistent evaluation of information. However, previous experiments demonstrating this effect retained two group-level processes, namely (a) social validation of information supporting the group members' initial preferences and (b) presentation of the additional information in a discussion format. Therefore, it was unclear whether the individual preference effect depends on the co-occurrence of these grouplevel processes. Here, we report two experiments demonstrating that the individual preference effect is indeed an individual-level phenomenon. Moreover, by a comparison to real interacting groups, we can show that even when all relevant information is exchanged and when no coordination losses occur, almost half of all groups would fail to solve hidden profiles due to the individual preference effect.
\end{abstract}

\section{Keywords}

group decision-making, hidden profiles, individual preference effect, preference-consistent evaluation of information, social validation

Paper received 25 April 2009; revised version accepted 3 March 2010.

Hidden profiles are group-decision tasks in which the correct solution cannot be detected by an individual group member prior to the group discussion, because each member possesses a subset of information supporting an inferior decision alternative (Stasser \& Titus, 1985). This is due to the distribution of shared and unshared information across individuals: shared information is available to all group members prior to discussion, whereas unshared information is uniquely held by one member only.
In the case of hidden profiles, shared and unshared information have different decisional implications,

\footnotetext{
${ }^{1}$ Georg-August-University Göttingen

${ }^{2}$ Ludwig-Maximilians-University Munich

Corresponding author:

Nadira Faulmüller, Georg-August-University Göttingen, Institute of Psychology, Economic and Social Psychology Unit, D-37073 Göttingen, Germany. [email: faulmueller@psych.uni-goettingen.de]
} 
and the alternative implied by the unshared information is the correct one. Hidden profiles therefore represent the prototype of situations in which groups have the potential to outperform individual decision-makers. Unfortunately, groups typically fail to solve hidden profiles. In most cases, they choose the alternative implied by their members' shared information (for reviews, see Brodbeck, Kerschreiter, Mojzisch, \& Schulz-Hardt, 2007; Kerr \& Tindale, 2004; Mojzisch \& Schulz-Hardt, 2006; Stasser \& Birchmeier, 2003).

\section{Group-level explanations for the failure of groups to solve hidden profiles}

Two group-level processes have been proposed to account for the failure of groups to solve hidden profiles: according to one explanation, groups do not discuss sufficient unshared information for the superiority of the correct solution to become evident (Stasser, 1992). This dominance of shared information has been attributed to the simple fact that shared information can be mentioned by each group member whereas unshared information, by definition, is known by only one member and therefore cannot be discussed if this member fails to mention it (i.e., there is a probabilistic sampling advantage for shared information; Stasser, 1992). Other authors have traced the dominance of shared information back to the fact that shared, but not unshared, information can be socially validated by other group members during discussion (Parks \& Cowlin, 1996). Social validation refers to learning that others in the group possess the same information and therefore can attest to its accuracy. As proposed by Wittenbaum, Hubbell, and Zuckerman (1999), social validation fuels a group's tendency to repeat previously mentioned shared information. In addition, there is evidence that social validation increases the perceived accuracy and relevance of information which, in turn, increases the decisional impact of information (Mojzisch, Schulz-Hardt, Kerschreiter, Brodbeck, \& Frey, 2008).

According to the second explanation, group members primarily use the discussion to negotiate their preferences rather than to consider the relevant information (Gigone \& Hastie, 1993). In the case of a hidden profile, this negotiation focus is likely to lead to a premature consensus on a suboptimal alternative, because the hidden profile distribution of information implies suboptimal individual preferences at the beginning of the discussion and, therefore, the correct solution has hardly any proponents in the negotiation process.

\section{The individual preference effect}

Greitemeyer and Schulz-Hardt (2003) proposed an alternative explanation for the failure of groups to solve hidden profiles, the individual preference effect. They argued that even in the absence of any dysfunctional group-level processes, the development of a suboptimal prediscussion preference leads to biased evaluation of the information exchanged during discussion: information supporting the group members' prediscussion preferences (preference-consistent information) is perceived to be of higher quality than information contradicting those preferences (preferenceinconsistentinformation). Because of this evaluation bias, group members tend to stick to their initial suboptimal preferences even if all unshared information is discussed and no premature group consensus is reached. It should be noted that the reason for this evaluation bias need not be some sort of defensive avoidance, that is, an intentional distortion of information. Rather, a biased evaluation in favor of members' prediscussion preferences could simply be a consequence of different amounts of cognitive resources being allocated to preference-consistent versus preferenceinconsistent information: as outlined by Edwards and Smith (1996), people might use the inconsistency between their prior beliefs and the newly learned preference-inconsistent information as a cue that either this information or their prior belief could be false, and therefore instigate a more detailed analysis of this piece of information. Conversely, the match of preference-consistent information with prior beliefs indicates that this piece of information can be accepted without much further testing. As a consequence of the more intensive testing of preference-inconsistent 
information, group members are more likely to detect weaknesses in it, making preferenceinconsistent information appear to be of lower quality on average than preference-consistent information (cf. Greitemeyer \& Schulz-Hardt, 2003). Empirical support for this idea can be found in research on motivated reasoning (e.g., Ditto \& Lopez, 1992; Ditto, Scepansky, Munro, Apanovitch, \& Lockhart, 1998) and the prior-belief effect (e.g., Edwards \& Smith, 1996).

In the experiments by Greitemeyer and SchulzHardt (2003), participants first received incomplete information about a decision task which was either representative of the entire information set (manifest profile; ${ }^{1}$ Lavery, Franz, Winquist, \& Larson, 1999) or was not (hidden profile). Next, instead of discussing the decision case with each other, participants individually read a fictitious discussion protocol containing full information exchange but with no group decision being reached. They were asked to rate the importance, accuracy, and valence of each piece of information and to reach a final individual decision. The results showed that few participants solved the hidden profile. Preference-consistent information was judged to be of higher quality than preferenceinconsistent information, and this evaluation bias mediated the tendency of individuals to stick to their initial preferences. In sum, the individual preference effect impedes the solution of hidden profiles even if all information is exchanged and no preference negotiation takes place.

\section{The present research}

The present experiments aimed to provide a critical test for the idea that the individual preference effect in group decision-making is indeed an individuallevel phenomenon. At first glance, the experiments by Greitemeyer and Schulz-Hardt (2003) seem to provide unequivocal evidence for the prediction that groups fail to solve hidden profiles even in the absence of dysfunctional group-level processes. However, a closer look reveals that their experimental setup still contained two group-level processes.

First, the misleading shared information was supported by social validation. As outlined in the first of the two Greitemeyer and Schulz-Hardt (2003) experiments, participants in the hidden profile condition were less likely to detect the best candidate than participants in the manifest profile condition. However, these two conditions differed not only with regard to the participants' initial preferences, but also with regard to which items were socially validated in the discussion protocol: each time a shared item was mentioned by a group member, the protocol contained a passage about the other members confirming that they held the same information. Since most of the shared items supported the correct candidate in the manifest profile condition but an incorrect candidate in the hidden profile condition, preference consistency and social validation of information were partially confounded. As research has demonstrated that social validation increases the perceived quality of information (Greitemeyer, Schulz-Hardt, \& Frey, 2003; Mojzisch, Grouneva, \& Schulz-Hardt, in press; Mojzisch et al., 2008; Postmes, Spears, \& Cihangir, 2001; Wittenbaum et al., $1999^{2}$ ), the failure of individuals to solve the hidden profile could be a consequence of social validation rather than a consequence of the participants' initial preferences. In their second experiment, Greitemeyer and Schulz-Hardt showed that suboptimal individual preferences hinder the solution of hidden profiles over and above any social validation effects. In other words, when social validation is present, suboptimal individual preferences have an additional hindering effect on the solution of hidden profiles. However, they did not test for individual preference effects in the absence of social validation. Therefore, based on their results we do not know whether or not the individual preference effect is contingent upon the copresence of social validation.

Second, in the Greitemeyer and Schulz-Hardt (2003) study, the information was presented in a discussion format. Since the protocol used was designed to look like an authentic transcript of a discussion of a three-person group, some typical attributes of verbal social interaction were retained, such as whole sentences and filler words. Hence, the protocol included residues of group processes. Such residues could have complicated the detection of the correct solution. For instance, phrases containing decision-irrelevant information could have 
distracted some of the readers' attention from the decision-relevant information. Moreover, lack of structuring (i.e., running text instead of tallying pieces of information describing each candidate) could have made it more difficult for participants to relate the decision-relevant pieces of information to each other, which is necessary if the information pattern reflecting the quality of the decision alternatives described is to be identified (Voigtlaender, Pfeiffer, \& Schulz-Hardt, 2009). Somewhat related to this, Jonas, Schulz-Hardt, Frey, and Thelen (2001) have shown that preference-consistent information-processing is stronger if information is presented sequentially as compared to simultaneously, and a discussion is a prototype of a sequential information presentation format. Thus, it could be argued that the residues of group processes contained in the experimental material of Greitemeyer and Schulz-Hardt impeded the solution of the hidden profile compared to a situation without any features of a group discussion, for example, if the additional information had been presented as a simple list of items.

In sum, based on the experiments reported by Greitemeyer and Schulz-Hardt (2003) it would be premature to conclude that the individual preference effect is a purely individual-level process. Instead, it is possible that this effect requires the copresence of two group-level processes, namely (a) social validation of the information supporting the suboptimal individual preference, and (b) presentation of the full information exchange in a discussion format. Both of these group-level residues could make it more difficult to detect the correct solution. To resolve this ambiguity, in one experiment we tested for individual preference effects in the absence of social validation effects. In a second experiment, we eliminated all aspects of verbal social interaction from the simulation of full information exchange.

But even if we accept that the individual preference effect constitutes a purely individual-level explanation of why groups are unlikely to solve hidden profiles, it is unclear how much of the overall failure is due to the individual preference effect as compared to the group-level processes that have been previously discovered. As has been demonstrated in many studies, groups tend to focus on shared information during discussion and, therefore, omit the critical unshared information from discussion. Obviously, if the information necessary to detect the correct solution is not exchanged at all, groups will fail to solve a hidden profile independently of whether or not they asymmetrically evaluate the information in the light of their members' initial preferences. In other words, when placed alongside the other explanations for the inability of groups to solve hidden profiles, the individual preference effect may only have a relatively weak effect. We cannot determine the relative importance of the individual preference effect as no data are available on this issue. As a first step towards rectifying this situation, in Experiment 2 we tested to what extent it is necessary to refer to group processes to explain the empirically observed low solution rates of real interacting groups.

\section{Experiment 1}

The aim of Experiment 1 was to determine whether the individual preference effect impedes the solution of hidden profiles in the absence of social validation of information supporting the group members' individual suboptimal preferences. The experimental procedure mostly followed that of Greitemeyer and Schulz-Hardt (2003; Experiment 1). Participants received individual information about three job candidates A, B, and C, and were asked to indicate their decision preference for one of them. Subsequently, participants read the protocol of a fictitious discussion they supposedly had had with two other group members and then made their final choice. The discussion protocol contained all available information, revealing Candidate $\mathrm{A}$ as the best choice. The information contributed by the fictitious other group members in the protocol predominantly supported the suboptimal Candidate B. Consequently, Candidate B was supported by shared (i.e., socially validated) information in all experimental conditions. The initial information assigned to the participants varied: in condition Pref_B, the participants' prediscussion information (like that of their cogroup members) implied Candidate B was the best, and hence, the 
participants' suboptimal preference was supported by the socially validated information. Thus, this condition constituted a replication of the hidden profile conditions by Greitemeyer and SchulzHardt. In condition Pref_C however, the participants' initial information set implied an alternative that was suboptimal as well, but not supported by the arguments of their fictitious discussion partners, namely Candidate C. Note that in this condition, the tendency to stick to one's initial preference should lead participants to choose Candidate C after having read the discussion protocol, whereas a social validation effect (i.e., reliance on shared information) should lead participants to select Candidate B. As a control, the prediscussion information given in condition Pref_A made the best choice (Candidate A) evident right away (manifest profile condition).

If we are correct in assuming that the individual preference effect does not require social validation of information supporting the initially preferred candidate, suboptimal individual preferences should affect the final decision even if full information about the alternative is given after the initial preference information. In particular, if most of the participants in condition Pref_C stick to Candidate $\mathrm{C}$ even though the socially validated information favors Candidate B, we can conclude that the individual preference effect does not require the co-occurrence of social validation and thus is sufficient to cause most or all participants to stick to their original preference.

\section{Method}

Participants and design The sample included 90 students (65 female, 25 male; mean age $=24$ years) who were offered refreshments in exchange for participation. The experiment had a one-factorial between-subjects design with three conditions in which a preference was induced either for Candidate A, B, or C: Pref_A versus Pref_B versus Pref_C.

Materials We adopted a decision case already used in a group experiment reported by Brodbeck, Kerschreiter, Mojzisch, Frey, and Schulz-Hardt
(2002). The materials consisted of summary descriptions of three hypothetical candidates applying for a professorship. The full information set comprised 35 attributes which were positive (e.g., "The candidate is seen as helpful to others"), neutral (e.g., "The candidate is 34 years old”), or negative (e.g., "The candidate does not allow interruptions of any kind during lectures"). The full candidate information consisted of 12 attributes for each candidate (with the neutral information item "The candidate is married" being used for two candidates) and implied Candidate A to be the best decision (eight positive, one neutral, and three negative items), followed by Candidates B and C (five positive, four neutral, and three negative items, each). The pretest results regarding the candidates' attributes and the information distribution are reported in the article by Brodbeck et al. (2002).

In all three experimental conditions, participants were given only partial information prior to reading the discussion protocol. Out of the full information set, participants in condition Pref_A initially received a subset of items describing $A$ as the best candidate (seven positive, one neutral, no negative), superior both to Candidate B (five positive, two neutral, one negative) and Candidate $\mathrm{C}$ (three positive, four neutral, one negative). In condition Pref_B, the items in the information subset favored Candidate B (five positive, two neutral, one negative), followed by Candidate $\mathrm{C}$ (three positive, four neutral, one negative) and Candidate A (four positive, one neutral, three negative). In condition Pref_C, Candidate $\mathrm{C}$ was the best initial choice (five positive, three neutral, no negative items), followed by Candidates $\mathrm{A}$ and B (four positive, one neutral, three negative items each). For every item subset, the superiority of the respective candidate was confirmed by a pretest with a total of 60 students. In condition Pref_A, Candidate A was judged by $89 \%$ of the participants as the best, followed by Candidates $\mathrm{B}$ and C (5\% each). In condition Pref_B, Candidate $\mathrm{B}$ was chosen by $76 \%$, followed by Candidate C $(14 \%)$ and Candidate A (10\%). And in condition Pref_C, Candidate $\mathrm{C}$ was picked by $85 \%$, followed by Candidate B (10\%) and Candidate A (5\%). 
The informational pattern of the subset assigned to the participants' two fictitious group members remained the same in all experimental conditions, arguing in favor of Candidate B. Thus, the two fictitious group members in all conditions received the identical information distribution as participants of condition Pref_B.

The participants were instructed to imagine that they were involved in the discussion. In the discussion protocol, the protagonists were labeled "You" (i.e., the group member who had received the same information set as the participant), "Person 1", and "Person 2". In all conditions, the protocol contained all 35 attributes overall available, and the wording and order of the information mentioned was identical. The preferences of the two fictitious group members (i.e., Person 1 and Person 2) were not mentioned. Different topics were discussed, one after the other. The information items were presented without evaluating comments and no inferences about which candidate might be the best were made. Unshared pieces of information were mentioned by the one group member who individually possessed this item and were not commented upon by the two others. When a shared argument was mentioned, the group members whose prediscussion information set also contained this item confirmed it. (An example is, "Person 2: 'Candidate $\mathrm{C}$ has one child.' Person 1 and you say that you also possess this information.”) Because Person 1 and Person 2 initially received a preponderance of information in favor of candidate $B$, the socially validated information supported B in all experimental conditions. Please note that since there was no information partially shared between Person 1 and Person 2 (i.e., shared by them but not by the participant) in the experimental conditions Pref_B and Pref_C, the items which were socially validated were always known by the participants prior to reading the discussion protocol. In other words, in both experimental conditions there was social validation of the participants' own information but no social validation of information new to the participant. Out of 12 shared items overall available in each experimental condition, four were first mentioned by the participant, four by Person 1, and four by Person 2. Thus, introduction of shared information into discussion and validation of this information by others was balanced across the three group members. The protocol ended after all items had been exchanged.

Procedure A cover letter outlined the aim of the study, namely an investigation into how teaching staff are assessed. Participants received the initial information set and were asked to indicate which candidate they preferred. Next, participants learned that decisions like the one at hand were often made by groups following a group discussion. To simulate such a situation, they would receive a protocol of a real group discussion. After that, the initial information set was collected before the protocol was handed out. As a consequence, as is typical in hidden profile studies, participants had to rely on their memory for the information previously read when receiving full information. Before reading the protocol, participants were also informed that the three group members acting in the protocol did not have identical information. However, based on the full information documented, one candidate was clearly better than the other two. The participants' task was to identify the best candidate. Successful participants would take part in a raffle to win one of 20 CD vouchers. After they had finished reading the protocol, participants were asked to make their final decision about which candidate was best. Finally, they were debriefed and thanked for participating.

\section{Results and discussion}

Seven participants had to be excluded from statistical analyses. Three of them made ambiguous statements concerning their prediscussion preference, another three showed random answer behavior in large parts of the experiment and one guessed the purpose of the study. Of these seven participants, one was originally assigned to condition Pref_C, and three each to conditions Pref_A and Pref_B. A manipulation check showed that 
the experimental induction of the individual decision preferences was successful overall, with $90 \%$ of the participants choosing the candidate implied by their information subsets. Eight participants whose prediscussion choice differed from the preference induced were excluded from all analyses. This seems appropriate since we are interested in the conditions under which participants are unable to give up their suboptimal prediscussion preference when faced with full information. Obviously, if a participant favors the optimal solution right from the beginning and sticks to this solution, it would not be correct to say that this person had overcome the individual preference effect or the influence of social validation, respectively. Four of the participants excluded were originally assigned to condition Pref_B, two each to conditions Pref_A and Pref_C. However, the results of all significance tests reported below remained the same when all 90 participants were included.

\section{Decision quality}

Of all participants included in the analyses (75 participants), $45 \%$ chose candidate A after having read the discussion protocol and, therefore, made the correct choice. Thirty-one percent of the participants selected Candidate B as most suited and $24 \%$ chose Candidate C. The participants' final decisions significantly depended on the experimental condition, $\chi^{2}(4, N=75)=36.10, p<.001$. Post hoc tests revealed that in condition Pref_A (i.e., the manifest profile condition) the best alternative (Candidate $\mathrm{A}$ ) was chosen more frequently $(76 \%)$ than Candidates B $(16 \%), \chi^{2}(1, N=23)=$ $9.78, p<.005$, and $\mathrm{C}(8 \%), \chi^{2}(1, N=21)=13.76$, $p<.001$. Conversely, in condition Pref_B, most participants picked Candidate B $(58 \%)$ as the best, followed by participants choosing Candidates A $(38 \%), \chi^{2}(1, N=23)=6.40, p<.05$, and $C(4 \%), \chi^{2}(1, N=15)=11.27, p<.005$. We hence replicated the findings of Greitemeyer and Schulz-Hardt (2003; Experiment 1), who found that most participants in the manifest profile condition chose the best candidate, whereas most participants in the hidden profile condition selected the suboptimal candidate implied by their prediscussion information and additionally supported by socially validated information. In condition Pref_C, where the individual preference effect and the social validation effect had different decisional implications, Candidate C (58\%) was chosen more frequently than both Candidate A $(23 \%), \chi^{2}(1, N=21)=3.86, p<.05$, and Candidate B $(19 \%), \chi^{2}(1, N=20)=5.00, p<.05$. Thus, participants who preferred Candidate $C$ based on their prediscussion information predominantly stuck to their initial choice, even though the implication of the full information was Candidate A, and the implication of the socially validated information was Candidate $B$.

In general, final decisions were largely determined by the participants' prediscussion preferences, with $65 \%$ of the participants maintaining their initial choice, compared to $35 \%$ correcting it one way or the other. Therefore, the participants' initial preferences were significantly associated with their final choices as shown by a Chi-Square Test for independence, $\chi^{2}(4, N=75)=37.94, p<$ $.001 .^{3}$ A direct comparison showed that the percentages of participants sticking to their initial preference were exactly the same in conditions Pref_B and Pref_C (58\%, each), $\chi^{2}(1, N=30)=0$, $p=1$. Hence, the individual preference effect hinders the solution of hidden profiles even if information supporting the group members' initial preference is not supported by social validation.

Please note that a limitation of our findings is that we did not rotate the items across conditions. Therefore, the individual preference effect in Experiment 1 could (at least in part) be due to some systematic differences in the candidates' attributes. To rule out this possibility, we rotated the items across conditions in Experiment 2.

\section{Experiment 2}

As noted above, in the Greitemeyer and SchulzHardt (2003) study, the information was presented in a discussion format and, hence, some typical attributes of verbal social interaction were retained (e.g., whole sentences and filler words). As recently shown, the probability of solving a 
hidden profile is significantly lower when full information is simply presented as a protocol of a group discussion to be read rather than when listed and structured by the participants as they read through the protocol (Voigtlaender et al., 2009). This implies that presenting the information in a discussion format makes it harder for the participants to integrate the new information, thereby promoting the individual preference effect. Arguably, such residues of group processes as found in the Greitemeyer and SchulzHardt experiments may have hampered the solution of the hidden profile compared to a situation without any features of a group discussion. In other words, we cannot rule out that the low solution rates found by Greitemeyer and SchulzHardt and also in our Experiment 1 were at least partially due to the specific format used to present the pieces of information.

Therefore, the first goal of Experiment 2 was to provide evidence for the individual preference effect in an experimental setting freed as much as possible from residues of group interaction. Hence, in Experiment 2, we did not use discussion protocols. Instead, full information was presented to individuals in the same manner as it is given to groups prior to interaction in typical hidden profile experiments (e.g., Brodbeck et al., 2002; Scholten, van Knippenberg, Nijstad, \& De Dreu, 2007; Schulz-Hardt, Brodbeck, Mojzisch, Kerschreiter, \& Frey, 2006). Specifically, participants in Experiment 2 received not only their own initial information set but also the sets assigned to their two hypothetical fellow group members in the form of one-page bulletpointed item lists. Thus, as participants read their own initial item list and the two "original" item lists of their (fictitious) cogroup members, all items were presented in an analogous manner. This made it possible to provide the participants with full information without resorting to a verbal communication format.

However, even if we were to find evidence for the idea that the individual preference effect causes groups to fail at hidden profiles in the absence of any group-interaction residues, it would still be unclear to what extent it would be necessary to refer to group processes to explain the empirically observed low solution rates of real interacting groups. Therefore, the second goal of Experiment 2 was to compare the performance of individuals working on a hidden profile task to the performance of real interacting groups.

To achieve these two goals, four experimental conditions were applied. In the hidden profile/individual condition, participants received a candidate information list supporting a suboptimal alternative and were asked to indicate their preference. Thereafter, they received the two remaining item lists and made their final choice based on all three lists. This is the psychological equivalent of a group member experiencing perfect information exchange in the group: the person enters the group with her/his own prediscussion information and, without any loss or any detracting elements, learns what the other group members know. In the manifest profile condition, no suboptimal initial preference was induced. Instead, participants received a onepage list containing all available information and were asked to make a decision after having read this list. We predicted that participants in the latter condition are more likely to detect the correct solution than participants in the first condition. Note, however, that a difference in the solution rates between these two experimental conditions could be due to both the individual preference effect and the simple fact that in the hidden profile/individual condition the information supporting the participants' initial preferences appeared on all three information lists and thus was repeated, which could lead to a higher decision impact of these items. To distinguish between these two possibilities, we included the no-preference induction/individual condition, in which participants received all three information lists at once, without making a preliminary choice based on a single list. Therefore, no initial preference was formed that could cause an individual preference effect, and differences in the results hence can be attributed exclusively to the repetition of information supporting the participants' preferred choice. 
To answer our second research question, namely to what extent it is necessary to refer to group processes to explain the empirically observed low solution rates of real interacting groups, in Experiment 2 we also included a realgroup hidden profile condition and compared the solution rates in this condition with the corresponding individual hidden profile condition. To this end, with the bidden profile/group condition we employed a condition that is typically used in hidden profile research: after having individually received their initial information and having indicated their candidate preference, participants were asked to discuss the decision case in a threeperson group and reach a unanimous decision. In this condition the group members collectively received exactly the same item lists as the participants in the hidden profile/individual and the nopreference induction/individual conditions. Note that in the hidden profile literature it is usual to compare the solution rate of groups who worked on a hidden profile to groups who worked with full information, that is, an extreme form of a manifest profile (e.g., Brodbeck et al., 2002; Lavery et al., 1999; Mojzisch \& Schulz-Hardt, 2010; Scholten et al., 2007; Schulz-Hardt et al., 2006). Therefore, after having made their individual decision, participants in the manifest profile condition were also assigned to three-person groups and additionally made a unanimous decision comparable to the decisions of the hidden profile groups. Thus, in the manifest profile condition both individual-level and group-level data were collected.

\section{Method}

Participants and design The sample included 95 students $(39$ female, 56 male; mean age $=27$ years), who were offered refreshments in exchange for participation. We employed a onefactorial between-subjects design with four conditions: hidden profile/individual (17 individual participants) versus manifest profile (15 individual participants) versus no-preference induction/ individual (18 individual participants) versus hidden profile/group (15 three-person groups).
Materials Similar to Experiment 1, materials consisted of attributes characterizing three candidates for a professorship. The full information set comprised 45 items. These items were selected from a pool of 153 positive, neutral, and negative pieces of information which had been rated for their valence and relevance with regard to the job as a professor in a first pretest with 98 participants (prior to this experiment). Full information described Candidate $\mathrm{A}$ as the best choice (nine positive, three neutral, three negative items), Candidate $\mathrm{C}$ as second best (six positive, six neutral, three negative items), and Candidate B as least suitable (six positive, three neutral, six negative items). A second pretest with 52 students had confirmed that this information distribution was clear as $87 \%$ of the participants chose Candidate A, 9\% Candidate C, and 4\% Candidate B.

The information distribution used to create a hidden profile was identical to the distributions in the experiments reported by Greitemeyer and Schulz-Hardt (2003) and Greitemeyer, SchulzHardt, Brodbeck, and Frey (2006). For this hidden profile information distribution (used in all conditions besides the manifest profile), the total items available were divided between three onepage information lists with nine attributes per candidate and list. Each list was designed to imply Candidate $\mathrm{B}$ as the best choice (six positive, one neutral, two negative items), and Candidates $\mathrm{C}$ (four positive, two neutral, three negative items) and $\mathrm{A}$ (three positive, three neutral, three negative items) as less desirable. In a third pretest with 28 students, Candidate B was selected as the best suited alternative by $83 \%$ of the participants choosing on the basis of the first information list, by $90 \%$ choosing on the basis of the second list, and by $60 \%$ choosing on the basis of the third list. Taken together, the three lists contained all available information on the Candidates A, B, and $\mathrm{C}$ with some of the items being shared and others being unshared.

In all experimental conditions, participants received full information before making the final choice, either individually or distributed between the members of a discussion group. Obviously, however, in the hidden profile/group condition 
unshared information of one group member was only available to the other group members if this member actually mentioned this piece of information during discussion.

Procedure In all conditions participants were told that they would work on a personnel-selection task and should make a decision about which of the three candidates should be hired. The experimenter emphasized that one of the candidates was clearly the best based on the full information and that cinema vouchers would be given to those who successfully identified this optimal choice.

The hidden profile/individual and the nopreference induction/individual condition differed only in the preliminary choice participants made before they read full information. In the hidden profile/individual condition, participants first received one of the information lists labeled " $\mathrm{X}$ " and introduced as "Your information about the three candidates". They were asked to study the candidate descriptions and to document their preference on a separate questionnaire sheet. Then, participants additionally received the two remaining lists labeled " $Y$ " and " $Z$ " and introduced as "Information of colleague $Y$ " and "Information of colleague Z", respectively. The lists $\mathrm{Y}$ and $\mathrm{Z}$ were described as information that two other colleagues had extracted from the candidates' applications. After having received all three lists and having been given as much time as needed to study these, participants were asked to make their final decision based on all available information.

In contrast, in the no-preference induction/ individual condition, participants made no initial choice. They were handed all three lists, X, Y, and $\mathrm{Z}$, together, introduced as "Information of colleague X", "Information of colleague Y", and "Information of colleague Z", the information that three colleagues had received about the job candidates. Participants were asked to make a decision about which candidate was best suited for the professorship based on all available information. In both conditions, we rotated which one of the three lists was labeled as either "X", "Y", or "Z", thus counterbalancing the order of presentation of lists.
In the hidden profile/group condition, each of the participants individually received one of the information lists $\mathrm{X}, \mathrm{Y}$, and $\mathrm{Z}$. In the manifest profile condition each participant was handed out a list with full information, beginning with information about $A$, then information about $B$, and then information about $\mathrm{C}$. Note that this full information list contained every piece of information only once (i.e., there were no repetitions). After having read this prediscussion information, participants were asked to indicate their individual preference for one of the candidates. Next, the information sheets and questionnaires were collected and three-person groups were assembled (consisting of one $\mathrm{X}_{-}$, one $\mathrm{Y}$-, and one Z-member in the hidden profile/group condition). Each group was instructed to discuss the decision case and make a unanimous decision. After the final decision was made, the experiment was over. Participants were debriefed and thanked for participation.

\section{Results and discussion}

One participant originally assigned to the hidden profile/individual condition guessed the purpose of the study and was excluded from all statistical analyses. However, including this person did not change the results. ${ }^{4}$ A manipulation check showed that the case material successfully induced a hidden profile, with Candidate B being the initial preference of most participants in the hidden profile/group (91\%) and the hidden profile/ individual (94\%) condition.

\section{Decision quality}

When comparing all individual conditions, decision quality significantly differed across conditions, $\chi^{2}(3, N=64)=28.89, p<.001$ (see Figure 1). Post hoc comparisons showed that participants in the hidden profile/individual condition found the correct solution significantly less frequently $(25 \%)$ than both individuals assigned to the manifest profile condition $(87 \%), \chi^{2}(1, N=31)=$ $11.89, p<.005$, and participants in the nopreference induction/individual condition (78\%), 


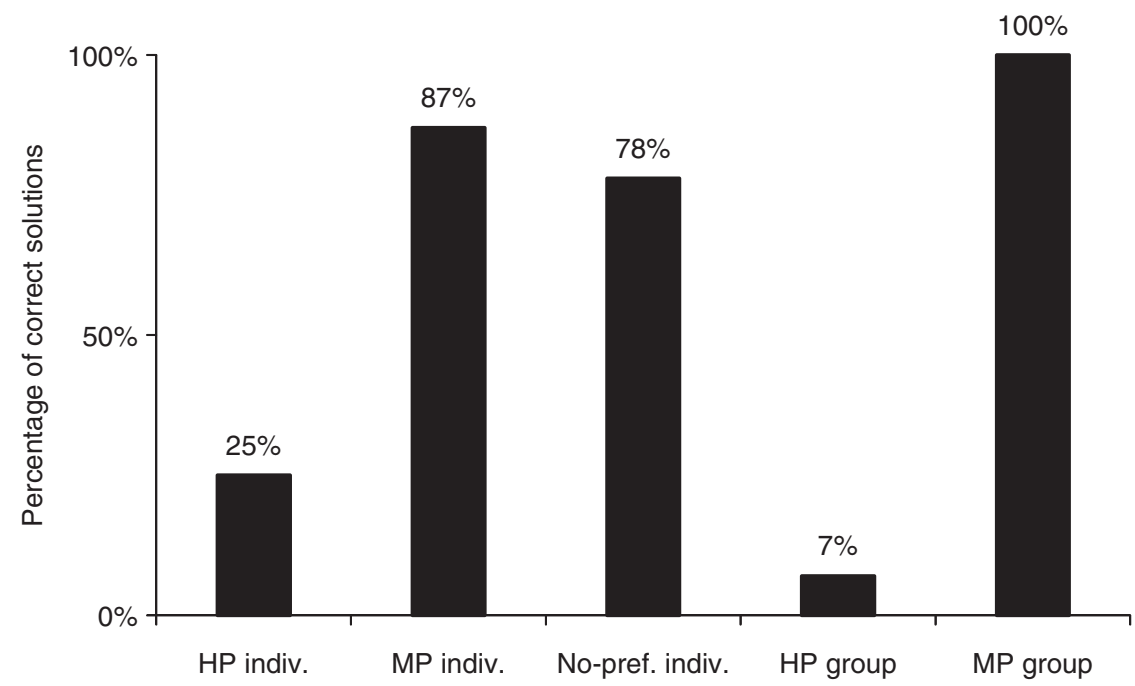

Full name of conditions and summary of the steps to reach the decision:

HP indiv. Hidden profile/individual

List $\mathrm{X} \rightarrow$ preference $\rightarrow$ lists $\mathrm{Y}$ and $\mathrm{Z} \rightarrow$ decision

MP indiv. Manifest profile/individual

Full information $\rightarrow$ decision

No-pref. indiv. No-preference induction/individual

Lists $\mathrm{X}, \mathrm{Y}$, and $\mathrm{Z} \rightarrow$ decision

HP group Hidden profile/group

List $\mathrm{X} \rightarrow$ preference $\rightarrow$ group discussion $\rightarrow$ decision

MP group Manifest profile/group

Full information $\rightarrow$ preference $\rightarrow$ group discussion $\rightarrow$ decision

Figure 1. Decision quality dependent on information distribution and level of decision-making in Experiment 2.

$\chi^{2}(1, N=34)=9.47, p<.01$, with the latter two conditions not significantly differing from each other, $\chi^{2}(1, N=33)=.453, p=.665$. In other words, we found that participants with suboptimal initial preferences were less likely to choose the correct candidate than participants without such preferences, thereby providing evidence for an individual preference effect.

As described above, after having stated their individual decision based on full information, participants in the manifest profile condition were assembled into three-person groups. When the final choices made in groups were compared, we noted that all manifest profile groups $(100 \%)$ had found the correct solution, compared to only 1 out of $15(7 \%)$ of the hidden profile groups, $\chi^{2}(1, N=20)=15.56, p<.001$ (see Figure 1). This replicates the consistent finding that groups typically fail to solve hidden profiles (e.g., Stasser \& Titus, 1985).

A comparison of the performance of groups to the performance of individuals in the hidden profile conditions revealed that 1 out of $15(7 \%)$ groups and 4 out of $16(25 \%)$ individuals solved the hidden profile. This difference is not significant, $\chi^{2}(1, N=31)=1.92, p=.333$. Please note 
that, in contrast to Experiment 1, we rotated the information across conditions in Experiment 2. Therefore, we can exclude systematic differences in the candidates' attributes being responsible for the individual preference effect.

Three possible limitations of our findings should be mentioned at this point. First, it is conceivable that floor effects or low test power led to Type II errors. This might, for example, have happened for the comparison between the hidden profile/group and the hidden profile/individual condition and, thus, the individual preference effect may not be sufficient to fully account for the failure of groups to solve hidden profiles.

Second, in the hidden profile/individual condition, the first information list, which provided the basis for forming the initial preference, was introduced to the participants as "Your information about the three candidates" whereas the first list in the no-preference induction/individual condition was labeled "Information of colleague X". As research regarding the so-called ownership bias (van Swol, Savadori, \& Sniezek, 2003) shows, people tend to evaluate own information more positively than information contributed by other group members (see also Chernyshenko, Miner, Baumann, \& Sniezek, 2003; Mojzisch et al., in press; van Swol et al., 2003). It is possible that participants in the hidden profile/individual condition experienced a higher degree of ownership regarding the initial information set. This could have contributed to the maintenance of suboptimal initial preferences in the hidden profile/individual condition. Mojzisch et al. (in press) showed that both ownership and preference consistency independently affect the evaluation of information. However, a comparison of the impact of these two characteristics on final decisions remains to be examined in further research.

Third, it should be explicitly noted that shared information appeared on all three item lists X, Y, and Z. Therefore, participants in the hidden profile/individual condition and the no-preference induction/individual condition indirectly learned which of the pieces of information from their original information set were also known by the two other group members. It is possible that these participants felt socially validated in some way. Hence, we cannot fully exclude that social validation supported the individual preference effect in the hidden profile/individual condition. However, as the solution rate for the hidden profile in the no-preference induction/individual condition is not significantly lower than in the manifest profile/individual condition, we can, at least, assume that potential social validation effects due to information repetition did not have a particularly strong impact.

\section{Real and potential group performance}

At first glance, the result that there is no significant difference in the solution rate of participants assigned to the hidden profile/individual condition compared to the hidden profile groups seems to imply that group-level processes are not necessary at all to explain the failure at hidden profile tasks. However, the explanatory power of this result is limited because we compared the decision quality of individuals with the decision quality of three-person groups. Yet, as the group performance literature suggests, when comparing individual to group performance, not the individual performance per se but its group-level equivalent should be used (cf. Steiner, 1972). To assess this so-called group potential, we have to answer the question of how hypothetical threemember groups that were only influenced by the individual preference effect but not by any dysfunctional group processes would have decided in our experiment. According to the results of the hidden profile/individual condition, such groups comprised, on average, $75 \%$ of members voting for a suboptimal alternative even though they received full information, and $25 \%$ of members who chose the correct alternative when faced with full information. Which decisions would such groups make? Basically, at least two different schemes can be applied to predict the decisions of such groups (cf. Davis, 1973).

If the majority model is applied, a three-person group would solve the hidden profile only if at least two of the three members favored the correct solution after the information exchange. In 
our case, the probability of two or three members proposing the correct solution and therefore choosing the best candidate is the sum of the probability of obtaining a group with two correct members and one incorrect member, $(0.25 \times 0.25$ $\times 0.75) \times 3=14 \%$, and the probability of obtaining a group with three correct members, $(0.25 \times 0.25$ $\times 0.25) \times 1=2 \%$. In other words, $16 \%$ of the groups applying this decision rule would solve the hidden profile. This hypothetical solution rate $(16 \%)$ is not significantly different from the actual solution rate of our hidden profile groups $(7 \%)$, $\chi^{2}(1, N=15)=.97, p=.324$. Therefore, if groups applied the majority model, the individual preference effect would be more or less sufficient to explain the empirically documented extent of the groups' failure. Even if no dysfunctional group processes were present at all, groups generally would fail to solve the hidden profile.

Ideally, however, groups follow the truth wins model. This model indicates that the correct solution, once proposed, is always adopted by the group. Thus, only a single group member would need to detect the best alternative to convince the other members of its superiority. In other words, the truth wins model implies that the agreement on the best alternative is made without any coordination losses within the group (Steiner, 1972). In a three-person group, the probability of a single member proposing the correct solution is 1 $(0.75 \times 0.75 \times 0.75)=58 \%$. Given the truth wins model, the hypothetical solution rate of a group only influenced by the individual preference effect therefore is $58 \%$, which is significantly higher than the empirical solution rate of the groups in the hidden profile/group condition $(7 \%), \chi^{2}(1, N=15)=16.22, p<.001$. However, this result implies that groups-even if they followed an ideal decision scheme and even if no impeding group processes were present at allwould only find the correct solution in somewhat more than half of all cases.

\section{General discussion}

For 25 years, the failure of groups to solve hidden profiles has been predominantly explained by group-level processes (for a review, see Stasser \& Birchmeier, 2003). Greitemeyer and Schulz-Hardt (2003) proposed an alternative perspective, the individual preference effect. According to this approach, groups fail to solve hidden profiles because their members tend to stick to their erroneous initial preferences. However, previous evidence for the individual preference effect came from experimental settings which still included two group-level processes, namely (a) social validation of the information supporting the participants' suboptimal preference, and (b) presentation of the additional information necessary to solve the hidden profile in a discussion format (Greitemeyer \& Schulz-Hardt, 2003).

The present study aimed to provide more clear-cut evidence for the idea that the individual preference effect is indeed a purely individuallevel phenomenon which does not depend upon the co-occurrence of group-level processes. In Experiment 1 we sought to disentangle the individual preference effect and the effect of social validation. Our results demonstrate that when the individual preference effect and social validation of the participants' own information lead to contradictory predictions, the individual preference effect prevails: participants maintained their initial preference even though the socially validated information favored another alternative. Hence, the individual preference effect hinders the solution of hidden profiles even if information supporting the group members' initial preference is not supported by social validation.

In Experiment 2, we aimed to provide evidence for the individual preference effect in an experimental setting freed as much as possible of features of verbal social interaction. Therefore, in contrast to previous research, we did not use a discussion protocol to simulate information exchange (cf. Greitemeyer \& Schulz-Hardt, 2003), but provided full information on simple, bulletpointed item lists. As predicted, participants with suboptimal initial preferences were less likely to solve a hidden profile than participants without such preferences. The individual preference effect hinders the detection of a hidden profile's best choice, even if not supported by a discussion-like 
presentation format or repetition of misleading information. In sum, Experiments 1 and 2 provide first-time evidence for the idea that the individual preference effect is an individual-driven process that is capable of impeding the solution of hidden profiles, even when efforts have been made to eliminate hindering group processes as far as possible.

Still the question remains as to how much of the overall failure of groups to solve hidden profiles is due to the individual preference effect as compared to the group-level processes proposed in previous research. We addressed this question in Experiment 2 by directly comparing the performance of individuals to the performance of three-person groups working on exactly the same hidden profile task. Our results indicate that if groups follow the common decision scheme of majority rule (Davis, 1973), the individual preference effect is sufficient to fully explain the empirically documented extent of the failure of groups to solve hidden profiles. Ideally, however, groups follow the truth wins model to combine the diverging opinions of their members; that is, they adopt the correct solution even if it is only proposed and defended by a single group member (cf. Laughlin \& Ellis, 1986). In this case, our results showed that the hypothetical solution rate of groups only influenced by the individual preference effect is $58 \%$. In other words, even if groups follow the truth wins model, the individual preference effect is sufficient to explain almost half of the failure of groups to solve hidden profiles. In sum, if only the individual preference effect was active, even when all relevant information is exchanged and when an ideal decision scheme applies, still almost half of all groups would fail to solve a typical hidden profile task-provided no additional support is given to these groups, for example, by introducing a successful group discussion technique (cf. Brodbeck et al., 2007). More generally, it is important to note that the individual preference effect can explain why groups may fail to solve hidden profiles even if the information exchanged is sufficient to allow the solution to be detected (Dennis, 1996; Greitemeyer et al., 2006). Therefore, one important lesson that can be learned from this research is that in case of hidden profiles, increasing the exchange of unshared information may not be sufficient to guarantee high decision quality. Instead, interventions that aim to facilitate the solution of hidden profiles also have to counteract the individual group members' tendency to stick to their initial incorrect preferences.

\section{Limitations}

It is possible that the content of discussion may have more impact on group members' final decisions in real interacting groups than it has on the final choice of individuals working alone. However, research suggests that the individual preference effect also operates in interacting groups, since preference-inconsistent information is devalued by group members interacting face-to-face (Klocke, 2007; Mojzisch et al., 2008; Toma \& Butera, 2009; van Swol, 2007). Moreover, it has been shown that even if an advocacy group decision procedure is applied that precludes members from prematurely aggregating their preferences and that leads to more comprehensive information pooling, group decisions are not improved in hidden profile situations (Greitemeyer et al., 2006), which could be due to the individual preference effect. Also, a recent study has revealed that even if information items are used that allow a direct comparison of decision alternatives and thus should facilitate the solution of the hidden profile, group members mostly did not integrate information contradicting their prediscussion preferences - although these groups did not sample more preference-consistent shared than preference-inconsistent unshared information (Reimer, Kuendig, Hoffrage, Park, \& Hinsz, 2007). Nevertheless, investigating the individual preference effect in real groups to further elucidate its mode of operation represents a promising endeavor for future research.

We argue that in hidden profiles where sharedness and preference-consistency of information are confounded to some extent, it may often appear that people favor shared over unshared information, but the real driving force behind this phenomenon is preference-consistency. However, it is 
important to state explicitly that the individual preference effect does not rule out shared information having an evaluation advantage (Greitemeyer et al., 2003) and a discussion advantage (Stasser, 1992) over unshared information-independent of the information's preference-consistency. Hence, any effects that involve these "true" sharedness advantages are clearly beyond the scope of our preference-effect approach.

\section{Implications and future directions}

Our results imply that an investigation of individual-level processes in general and of the individual preference effect in particular is not only worthwhile, but also necessary to explain why groups fail to solve hidden profile tasks. Our proposition is in line with other recent studies suggesting that the failure of groups to solve hidden profiles may not only be a function of problems with group interaction, but also due to individual-level processes (see also Klocke, 2007; van Swol, 2007). Of course, this does not mean to say that we deny the existence of hindering group-level processes. Clearly, a group which fails to discuss the critical unshared information is unlikely to solve a hidden profile. Thus, we assume that the individual preference effect does not contradict but rather supplements existing group-level explanations. In fact, group-level processes might even moderate the operation of the individual preference effect (and vice versa). For instance, it is conceivable that in situations in which group members unanimously prefer an incorrect alternative, the individual preference effect could be even stronger than when individuals work alone. To test for possible moderating influences, future research could separately manipulate whether or not certain group processes can occur (e.g., by discussion format) and whether or not individual preferences were formed.

As hidden profiles represent the prototype of situations in which groups have the potential to outperform individuals in terms of decision quality, many researchers have developed interventions aimed at enabling groups to effectively handle hidden profile situations (e.g., Galinsky \&
Kray, 2004; Greitemeyer et al., 2006; Hollingshead, 1996; Klocke, 2007; Larson, Christensen, Franz, \& Abbott, 1998; Scholten et al., 2007; Stasser, Stewart, \& Wittenbaum, 1995). However, most interventions have focused on the dominance of shared information or premature preference negotiation, and thus on the insufficient exchange of unshared information (for exceptions, see Mojzisch \& Schulz-Hardt, 2010; Postmes et al., 2001). Interestingly, Gigone and Hastie (1993) did report that even if group members pooled sufficient information to solve a hidden profile, groups still failed to integrate the unshared cues. Unfortunately, they did not give any reasons for the failure of unshared information to significantly influence the group decision. The individual preference effect can provide an explanation: group members stick to their initial preferences because they evaluate the information exchanged in the light of these preferences. The unshared and thus preference-inconsistent information is systematically devalued and therefore barely taken into account when making the group decision. In line with the observation made by Gigone and Hastie (1993), most interventions focusing on the exchange of unshared information have not been successful in enhancing group decision quality (cf. Stasser \& Birchmeier, 2003). As our findings imply, group-level interventions-even assuming that they eliminated all dysfunctional group-level processes entirely — can only improve decision quality in little more than half of the cases at best. At worst, interventions do not lead to any improvements at all, as long as the impeding effects on the individual level are not taken into account. In conclusion, we believe it is promising to take a closer look at mediating and moderating factors of the individual preference effect. This would not only enrich group decisionmaking theory, but also form a solid basis for the development of effective interventions facilitating the solution of hidden profile tasks. Some interesting suggestions may be taken from recent literature. For example, Scholten et al. (2007) found that a high degree of epistemic motivation leads to an effortful analysis of the information exchanged in group discussions. It would be 
interesting to investigate whether a high degree of epistemic motivation also has an attenuating effect on biased evaluation of information. Furthermore, it would be interesting to examine if the individual preference effect is moderated by whether the group members' preferences are based on attractions toward alternatives, on aversions to alternatives, or on combinations of both. This difference has recently been found to have a strong impact on information processing in groups (Nijstad \& Kaps, 2008). Moreover, given prior work on the importance of minority dissent in improving the solution rates in hidden profile tasks (e.g., Schulz-Hardt et al., 2006), it might be interesting to explore in more detail how the individual preference effect is moderated by the constellation of the decision preferences of the group members. Finally, it is important to note that in everyday decision-making groups, individuals typically face a mixture of cooperative incentives to reach high-quality decisions and competitive incentives to reach their personal goals (De Dreu, Nijstad, \& van Knippenberg, 2008; Wittenbaum, Hollingshead, \& Botero, 2004). Recent research shows that when in competition with each other, group members are more reluctant to disconfirm their initial preferences compared to when cooperating with each other (Toma \& Butera, 2009). This finding tentatively indicates that competition might increase the individual preference effect. Since pressure to compete with others is commonplace in organizational and political settings, this finding highlights the importance of the individual preference effect in organizational and political decisionmaking groups.

To recap, there is no doubt that group-level processes play an important role in group decision-making: if groups fail to exchange the critical unshared information, it is unlikely that they will solve a hidden profile. Notwithstanding the importance of group-level processes, the present study shows that group-level processes do not tell the whole story, and that the individual preference effect is capable of impeding the solution of hidden profiles even if few or no hindering group-level processes are present.

\section{Acknowledgments}

We thank Norbert Kerr for his very helpful editorial comments and three anonymous reviewers for their constructive criticism of a subsequent draft. We also gratefully acknowledge the comments from Daniel May and Cecily French on an earlier version of this manuscript. Rudolf Kerschreiter and Andreas Mojzisch contributed equally to this article. Thus, an alphabetical order of authorship was chosen.

\section{Notes}

1. Note that by a manifest profile we mean any information distribution for which the participants' information set is representative of the entire information set. Under this definition, providing participants with full information from the beginning is but one (and the lengthiest) form of a manifest profile possible.

2. Note that in the studies of Postmes et al. (2001) and Wittenbaum et al. (1999), sharedness and preference consistency of information were partially confounded.

3. Of the eight participants excluded from the analyses because their prediscussion choice differed from the induced one, six $(=75 \%)$ maintained their initial preference.

4. In Experiment 2, a total of four participants assigned to the two hidden profile conditions (hidden profile/individual and hidden profile/group) initially preferred the correct candidate. Diverging from Experiment 1, these participants were all included in the statistical analyses in order to keep the experimental conditions comparable to each other. If we had excluded the one participant who preferred the correct candidate in the individual condition we would also have had to exclude all proponents of the correct solution in the group condition. In turn, this would have made it impossible to appropriately compare the real to the potential group performance, as the models for calculating the latter, which we apply and report here, are based on different distribution of preferences within the group.

\section{References}

Brodbeck, F. C., Kerschreiter, R., Mojzisch, A., Frey, D., \& Schulz-Hardt, S. (2002). The dissemination of critical, unshared information in decision-making groups: 
The effects of pre-discussion dissent. European Journal of Social Psychology, 32, 35-56.

Brodbeck, F. C., Kerschreiter, R., Mojzisch, A., \& SchulzHardt, S. (2007). Group decision making under conditions of distributed knowledge: The information asymmetries model. Academy of Management Review, 32, 459-479.

Chernyshenko, O. S., Miner, A. G., Baumann, M. R., \& Sniezek, J. A. (2003). The impact of information distribution, ownership, and discussion on group member judgment: The differential cue weighting model. Organizational Behavior and Human Decision Processes, 91, 12-25.

Davis, J. H. (1973). Group decision and social interaction: A theory of social decision schemes. Psychological Review, 80, 97-125.

De Dreu, C. K. W., Nijstad, B. A., \& van Knippenberg, D. (2008). Motivated information processing in group judgment and decision making. Personality and Social Psychology Review, 12, 22-49.

Dennis, A. R. (1996). Information exchange and use in small group decision making. Small Group Research, 27, 532-550.

Ditto, P. H., \& Lopez, D. F. (1992). Motivated skepticism: Use of differential decision criteria for preferred and nonpreferred conclusions. Journal of Personality and Social Psychology, 63, 568-584.

Ditto, P. H., Scepansky, J. A., Munro, G. D., Apanovitch, A. M., \& Lockhart, L. K. (1998). Motivated sensitivity to preference-inconsistent information. Journal of Personality and Social Psychology, 75, 53-69.

Edwards, K., \& Smith, E. E. (1996). A disconfirmation bias in the evaluation of arguments. Journal of Personality and Social Psychology, 71, 5-24.

Galinsky, A. D., \& Kray, L. J. (2004). From thinking about what might have been to sharing what we know: The effects of counterfactual mind-sets on information sharing in groups. Journal of Experimental Social Psychology, 40, 606-618.

Gigone, D., \& Hastie, R. (1993). The common knowledge effect: Information sharing and group judgment. Journal of Personality and Social Psychology, 65, 959-974.

Greitemeyer, T., \& Schulz-Hardt, S. (2003). Preferenceconsistent evaluation of information in the hidden profile paradigm: Beyond group-level explanations for the dominance of shared information in group decisions. Journal of Personality and Social Psychology, 84, 322-339.

Greitemeyer, T., Schulz-Hardt, S., Brodbeck, F. C., \& Frey, D. (2006). Information sampling and group decision making: The effects of an advocacy decision procedure and task experience. Journal of Experimental Psychology: Applied, 12, 31-42.

Greitemeyer, T., Schulz-Hardt, S., \& Frey, D. (2003). Präferenzkonsistenz und Geteiltheit von Informationen als Einflussfaktoren auf Informationsbewertung und intendiertes Diskussionsverhalten bei Gruppenentscheidungen Preference consistency and sharedness of information as predictors of information evaluation and intended behavior in group discussions]. Zeitschrift für Sozialpsychologie, 34, 9-23.

Hollingshead, A. B. (1996). The rank-order effect in group decision making. Organizational Behavior and Human Decision Processes, 68, 181-193.

Jonas, E., Schulz-Hardt, S., Frey, D., \& Thelen, N. (2001). Confirmation bias in sequential information search after preliminary decisions: An expansion of dissonance theoretical research on selective exposure to information. Journal of Personality and Social Psychology, 80, 557-571.

Kerr, N. L., \& Tindale, R. S. (2004). Group performance and decision making. Annual Review of Psychology, 55, 623-656.

Klocke, U. (2007). How to improve decision making in small groups: Effects of dissent and training interventions. Small Group Research, 6, 437-468.

Larson, J. R., Jr., Christensen, C., Franz, T. M., \& Abbott, A. S. (1998). Diagnosing groups: The pooling, management, and impact of shared and unshared case information in team-based medical decision making. Journal of Personality and Social Psychology, 75, 93-108.

Laughlin, P. R., \& Ellis, A. L. (1986). Demonstrability and social combination processes on mathematical intellective tasks. Journal of Experimental Social Psychology, 22, 177-189.

Lavery, T. A., Franz, T. M., Winquist, J. R., \& Larson, J. R., Jr. (1999). The role of information exchange in predicting group accuracy on a multiple judgment task. Basic and Applied Social Psychology, 21, 281-289.

Mojzisch, A., Grouneva, L., \& Schulz-Hardt, S. (in press). Biased evaluation of information during discussion: Disentangling the effects of preference 
consistency, social validation, and ownership of information. European Journal of Social Psychology.

Mojzisch, A., \& Schulz-Hardt, S. (2006). Information sampling in group decision making: Sampling biases and their consequences. In K. Fiedler \& P. Juslin (Eds.), Information sampling and adaptive cognition (pp. 299-325). Cambridge: Cambridge University Press.

Mojzisch, A., \& Schulz-Hardt, S. (2010). Knowing others' preferences degrades the quality of group decisions. Journal of Personality and Social Psychology, 98, 794-808.

Mojzisch, A., Schulz-Hardt, S., Kerschreiter, R., Brodbeck, F. C., \& Frey, D. (2008). Social validation in group decision making: Differential effects on the decisional impact of preference-consistent and preference-inconsistent information. Journal of Experimental Social Psychology, 44, 1477-1490.

Nijstad, B. A., \& Kaps, S. C. (2008). Taking the easy way out: Preference diversity, decision strategies, and decision refusal in groups. Journal of Personality and Social Psychology, 94, 860-870.

Parks, C. D., \& Cowlin, R. A. (1996). Acceptance of uncommon information into group discussion when that information is or is not demonstrable. Organizational Behavior and Human Decision Processes, 66, 307-315.

Postmes, T., Spears, R., \& Cihangir, S. (2001). Quality of decision making and group norms. Journal of Personality and Social Psychology, 80, 918-930.

Reimer, T., Kuendig, S., Hoffrage, U., Park, E., \& Hinsz, V. (2007). Effects of the information environment on group discussions and decisions in the hidden profile paradigm. Communication Monographs, 74, $1-28$.

Scholten, L., van Knippenberg, D., Nijstad, B. A., \& De Dreu, C. K. W. (2007). Motivated information processing and group decision making: Effects of process accountability on information processing and decision quality. Journal of Experimental Social Psychology, 43, 539-552.

Schulz-Hardt, S., Brodbeck, F. C., Mojzisch, A., Kerschreiter, R., \& Frey, D. (2006). Group decision making in hidden profile situations: Dissent as a facilitator for decision quality. Journal of Personality and Social Psychology, 91, 1080-1093.
Stasser, G. (1992). Pooling of unshared information during group discussion. In S. Worchel, W. Wood, \& A. Simpson (Eds.), Group process and productivity (pp. 48-67). Newbury Park, CA: Sage.

Stasser, G., \& Birchmeier, Z. (2003). Group creativity and collective choice. In P. B. Paulus \& B. A. Nijstad (Eds.), Group creativity (pp. 85-109). New York: Oxford University Press.

Stasser, G., Stewart, D. D., \& Wittenbaum, G. M. (1995). Expert roles and information exchange during discussion: The importance of knowing who knows what. Journal of Experimental Social Psychology, 31, 244-256.

Stasser, G., \& Titus, W. (1985). Pooling of unshared information in group decision making: Biased information sampling during discussion. Journal of Personality and Social Psychology, 48, 1467-1478.

Steiner, I. D. (1972). Group process and productivity. New York: Academic Press.

Toma, C., \& Butera, F. (2009). Hidden profile and concealed information: Strategic information sharing and use in group decision making. Personality and Social Psychology Bulletin, 35, 793-806.

Van Swol, L. M. (2007). Perceived importance of information: The effects of mentioning of information, shared information bias, ownership bias, reiteration, and confirmation bias. Group Processes \& Intergroup Relations, 10, 239-256.

Van Swol, L. M., Savadori, L., \& Sniezek, J. A. (2003). Factors that may affect the difficulty of uncovering hidden profiles. Group Processes \& Intergroup Relations, 6, 285-304.

Voigtlaender, D., Pfeiffer, F., \& Schulz-Hardt, S. (2009). Listing and structuring of discussion content as a means of improving individual decision quality in hidden profiles. Social Psychology, 40, 79-87.

Wittenbaum, G. M., Hollingshead, A. B., \& Botero, I. C. (2004). From cooperative to motivated information sharing in groups: Moving beyond the hidden profile paradigm. Communication Monographs, 71, 286-310.

Wittenbaum, G. M., Hubbell, A. P., \& Zuckerman, C. (1999). Mutual enhancement: Toward an understanding of the collective preference for shared information. Journal of Personality and Social Psychology, 77, 967-978. 


\section{Biographical notes}

NADiRA FAULMÜLLER is $\mathrm{PhD}$ student and Teaching Assistant in the Economic and Social Psychology Unit at the University of Göttingen. Her research mainly focuses on social information-processing and group decision-making.

RUDOLF KERSCHREITER is currently substitute Professor of Social Psychology at the Department of Psychology, Ludwig-Maximilians-University, Munich. His research interests include group decision-making, social information-processing, social identity, social exchange, and leadership.

ANDREAS MOJZISCH is a post-doc and resident scientist in the Economic and Social Psychology Unit at the University of Göttingen, and member of the Courant Research Centre "Evolution of
Social Behavior." His research interests include group decision-making, emergence of leadership in groups, social cognitive neuroscience, and occupational health.

STEFAN SCHULZ-HARDT is full Professor for Industrial, Economic and Social Psychology at the University of Göttingen and a principal investigator in the Courant Research Centre "Evolution of Social Behavior." His main research interests are group decision-making and group performance, social information search and processing, escalating commitment, and stress at the workplace. He has coauthored numerous articles in top journals in the field of social psychology as well as industrial and organizational psychology. Currently, he is Associate Editor of the European Journal of Social Psychology. 